\title{
L'HOMME L'Homme
}

Revue française d'anthropologie

$189 \mid 2009$

Oralité et écriture

\section{Les animaux, les hommes et l'Alliance}

Une lecture anthropologique de quelques textes bibliques

Jean-Pierre Albert

\section{OpenEdition}

Journals

Édition électronique

URL : http://journals.openedition.org/lhomme/21997

DOI : 10.4000/lhomme.21997

ISSN : 1953-8103

Éditeur

Éditions de l'EHESS

Édition imprimée

Date de publication : 1 janvier 2009

Pagination : 81-114

ISSN : 0439-4216

Référence électronique

Jean-Pierre Albert, "Les animaux, les hommes et l'Alliance », L'Homme [En ligne], 189| 2009, mis en

ligne le 01 janvier 2011, consulté le 19 avril 2019. URL : http://journals.openedition.org/

Ihomme/21997 ; DOI : 10.4000/lhomme.21997 


\title{
Les animaux, les hommes et l'Alliance Une lecture anthropologique de quelques textes bibliques
}

\author{
Jean-Pierre Albert
}

$\mathrm{L}^{\prime}$

OCCASION DE cette étude est la publication en France, à peu d'intervalle, des livres de Mary Douglas, L'Anthropologue et la Bible. Lecture du Lévitique ${ }^{1}$ (2004), et de Philippe Descola, Par-delà nature et culture (2005). Très éloignés à bien des égards, ils se rejoignent au moins sur un point: la réflexion sur les représentations et qualifications de la vie animale, avec un souci commun de décaler notre regard des "évidences " nourries aussi bien par ce que Philippe Descola appelle l'ontologie " naturaliste " que par l'image ordinaire d'une tradition judéo-chrétienne réduisant les animaux à un usage instrumental. Cette convergence mérite d'être analysée dans une double perspective. D'une part, comparer les hypothèses de Mary Douglas sur le statut de l'animal selon le Lévitique avec la théorie des quatre schèmes développée dans Par-delà nature et culture permettra d'examiner si la pensée de l'animal qu'elle met au jour ou, plus largement, celle que je tenterai de repérer dans d'autres textes bibliques, s'éclaire d'être rapportée à l'un au moins des modèles proposés. D'autre part, l'étude d'une tradition que Philippe Descola n'aborde qu'à travers son expression chrétienne peut, on va le voir, apporter à ses propositions théoriques une confirmation dont il ne semble pas avoir envisagé l'importance. Il considère en effet le christianisme (et sa lecture des premiers chapitres de la Genèse) comme une phase préparatoire à la domination du modèle naturaliste en Occident, et je lui donne jusqu'à un certain point raison ${ }^{2}$. Mais la tradition biblique et quelques-uns de ses prolongements

1. Traduction de Leviticus as Literature, New York, Oxford University Press, 1999.

2. Saint Augustin et saint Thomas d'Aquin sont invoqués comme représentants d'une version chrétienne de la chaine de l'être empruntée au néo-platonisme (Descola 2005 : 284). Le rôle du christianisme dans la constitution de l'idée moderne de nature est étudié pp. 102-105. 
dans les religions du Livre peuvent plutôt se comprendre dans la perspective de "l'analogisme" - un schème qui recouvre des systèmes religieux et/ou cosmologiques assez divers et, pour la plupart, extra-européens. Or sa présence dans le judaïsme ancien et diasporique comme dans certains aspects de la pensée chrétienne a sans doute eu plus de poids en Occident qu'à travers les constructions savantes où Descola le reconnaît - théories des signatures et de la grande chaîne de l'être. Mon propos n'est donc pas du tout de critiquer le modèle lui-même ou son applicabilité aux cas du judaïsme et du christianisme, mais au contraire de montrer qu'il offre une grille de lecture d'une grande pertinence. Il s'agira en somme d'ajouter une étude de cas à celles qui ont permis à Philippe Descola de construire son modèle : son absence du corpus de départ ayant une valeur intéressante de test si, comme je le pense, l'application de cette théorie permet de clarifier des aspects du texte biblique qui, sans cela, resteraient des énigmes.

En vérité, l'exercice que je propose est rendu difficile (et peut-être voué à n'aboutir qu'à des résultats incertains) en raison des problèmes que soulève préjudiciellement la notion même de «texte biblique ». On le sait, la Bible, y compris si l'on ne considère que ce que les chrétiens appellent l'Ancien Testament, est composite, juxtaposant des unités textuelles variées et, souvent jusqu'en leur sein - par exemple d'une phrase à l'autre au cours d'un récit - des strates rédactionnelles hétérogènes que les biblistes parviennent à identifier et à dater avec une probabilité toujours croissante. On est ainsi en présence d'un texte associant des productions intellectuelles échelonnées sur plusieurs siècles et renvoyant à des conceptions parfois incompatibles. Rien ne permet d'affirmer que l'on y trouvera, à propos de l'animal, une unité de vue qui fait défaut sur des questions aux enjeux autrement décisifs. De plus, le texte porte déjà la marque de relectures et interprétations que les commentaires juifs et chrétiens ont multipliées ensuite pendant près de deux millénaires, jusqu'à rendre l'original presque inséparable du sens que chaque époque lui a reconnu. Tel est, sans doute, le destin de tout texte sacré : ses obscurités appellent des "clarifications» qui sont autant de relectures souvent divergentes. Dans le cas du corpus biblique, ce phénomène est encore aggravé par le fait qu'il offre ses ressources fondatrices à trois religions soucieuses d'affirmer leur singularité.

Il reste cependant un texte dont la signification est pour partie dépendante des situations historiques et idéologiques que reflètent chacun pour son compte ses composants, mais qui a également pris la forme qu'on lui connaît parce qu'il était porteur de sens pour ceux qui ont eu la charge de le transmettre: moins de simples lecteurs-copistes passifs que des "arrangeurs » qui, au cours de la longue phase précédant sa fixation à peu près définitive, ont œuvré à lui donner un sens à leurs yeux acceptable et 
un certain degré de cohérence. Leurs interventions ont parfois ajouté encore au chaos, mais il faut aussi reconnaître que de larges pans de la Bible hébraïque - le Pentateuque et les livres historiques, par exemple se présentent comme des constructions très élaborées, et non comme un patchwork plus ou moins aléatoire de traditions hétéroclites ${ }^{3}$. Découvrir le sens et les intentions d'un texte biblique n'est donc pas un projet désespéré. Cette lecture peut être guidée par des recoupements internes et aussi par le recours à des traditions parallèles, parfois très anciennes, dont les écrits talmudiques et midrashiques se font l'écho. Il existe enfin le vaste corpus des écrits intertestamentaires et autres "apocryphes" anciens qui donnent accès à des lectures (interprétations des textes canoniques ou nouvelles productions par eux inspirées) antérieures à l'interprétation chrétienne comme à celle du judaïsme de la diaspora. Je distinguerai donc dans cette étude les matériaux bibliques (au sens large) de cette première époque des apports ultérieurs de commentaires et prolongements consti-

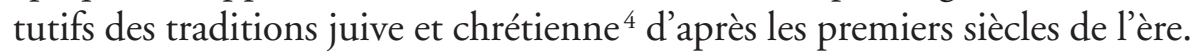

Ce long préalable méthodologique ne suffira sans doute pas à m’exempter des objections des spécialistes ${ }^{5}$. Il me permet toutefois de préciser mon hypothèse de travail. Le texte biblique, que je limiterai ici à la Bible hébraïque, comporte des références aux animaux que l'on peut ramener à trois grands types :

- des indications quant à leur origine, leur nature et leur place dans la création ;

- des énoncés normatifs précisant leurs usages par les hommes et le traitement qui doit leur être réservé ;

- des emplois métaphoriques à visée morale ou eschatologique que je n'étudierai pas car ils me semblent peu spécifiques: il serait abusif d'en inférer sans autre précaution une ontologie "analogiste " ou " animiste " sous-jacente ${ }^{6}$.

3. Les motivations idéologiques et l'artifice de ces constructions apparaissent d'autant plus si l'on accepte les rectifications historiques proposées, sur la base de l'archéologie, par Finkelstein \& Silberman (2002).

4. Le cas de l'islam, qui utilise des matériaux bibliques en dehors d'une reprise du texte lui-même, est un peu différent. Je ne l'aborderai pas dans cet article.

5. J'ai renoncé à étayer mon propos de références à l'immense érudition des études bibliques qui sont de règle lorsqu'on aborde ces textes, et j'assume à mes risques ce défaut évident de légitimité : en tout état de cause, ce que j'aurais pu produire serait resté très en-deçà des attentes en la matière. 6. Hegel, dans son Esthétique, distingue ainsi le statut des animaux dans les fables d'Ésope de celui qui leur est reconnu dans les religions orientales. Ésope le Phrygien, situé par sa naissance à la jointure de l'Orient et du monde grec, lui semble emblématique d'une position intermédiaire : l'utilisation désacralisée d'animaux pour incarner des dispositions morales qui ne peuvent être qu'humaines. 
Les sources des deux premiers types, même si elles restent d'une ampleur limitée, devraient être suffisantes pour spécifier l'existence dans la Bible hébraïque d'un schème ontologique dominant parmi ceux qu'a définis Philippe Descola. Nous savons aussi qu'elles ont été à l'origine des normes et représentations développées plus tard par le judaïsme et le christianisme. Or, comme on va le voir, les textes de référence sont assez obscurs ou ambigus pour soutenir des lectures plurielles, y compris à l'intérieur d'une même tradition religieuse. Ma démarche consistera donc d'abord à tenter de repérer dans les textes «nus » des indices de la présence structurante d'un schème, puis à montrer brièvement comment leurs relectures ont renforcé sa cohérence, ou l'ont au contraire effacée: une sélection orientée des sources, jointe à l'horizon intellectuel d'une tout autre période historique, suffit à suggérer une nouvelle interprétation. On verra ainsi comment le christianisme, au cours de son histoire, a recomposé à plusieurs reprises sa conception de l'animal sans jamais manquer d'appuis scripturaires! Avant d'entrer dans le vif du sujet, il me paraît souhaitable de résumer les aspects des propositions théoriques de Mary Douglas et Philippe Descola qui seront ici mobilisés.

\section{Un bref bilan}

L'œuvre scientifique de Mary Douglas semble marquée par sa confrontation, plusieurs fois remise sur le métier, avec le texte le plus aride du Pentateuque: le Lévitique. Sa lecture des "abominations» relatives aux animaux impurs, dans De la souillure, a fait date : elle montre en effet que, au lieu de rapporter leur exclusion de l'alimentation à des motifs (de longue date évoqués) hygiéniques ou écologiques (l'élevage du porc aurait des effets désastreux sur l'environnement), il suffit de suivre la lettre du texte. Les jugements d'impureté y sont explicitement motivés par le statut d'anomalie des espèces rejetées : soit des animaux comme le porc qui croisent des caractères typiques de groupes différents (les ruminants ont le sabot fendu, les non-ruminants ont le sabot rond ou n'ont pas de sabots du tout; le porc, lui, a le pied fendu mais n'est pas un ruminant); soit des animaux trop éloignés de la forme typique de leur classe (un animal aquatique doit être un poisson, pourvu d'écailles et de nageoires, pas un mollusque ou un crustacé). Le texte biblique n'en dit pas plus, mais Mary Douglas montre que des anomalies ou des formes de désordre de ce genre sont assez systématiquement associées, dans les cultures les plus diverses, aux notions de saleté ou d'impureté. Je dois dire que cette analyse, jointe à des considérations très analogues développées par Edmund Leach (1980), me paraît très satisfaisante. 
Tel n'est plus, cependant, l'avis de son auteure. Revenant sur cette question dans Leviticus as Literature, Mary Douglas est tout d'abord conduite à recadrer la question des animaux impurs : considérant le Lévitique comme une œuvre singulière, porteuse d'un enseignement sur certains points différent de celui du Deutéronome qui traite en grande partie des mêmes objets, elle envisage en des termes nouveaux la classification des animaux. Le Lévitique en effet opère, selon elle, une distinction systématique entre "impureté » et " abomination " absente du Deutéronome. Loin de concerner l'essence des réalités visées, ces catégories permettent l'une et l'autre de définir les obligations religieuses des Hébreux. La seconde en particulier, qui vise seulement des êtres «à éviter » et s'applique en fait à la plupart des espèces animales, marque bien la différence entre des êtres dépourvus en eux-mêmes de toute qualification négative et les conséquences résultant d'un contact avec eux ou, a fortiori, d'un usage alimentaire. Dieu n'a donc pas créé une classe d'êtres objectivement détestables, bien au contraire. Si l'on prend le cas des petites bêtes (vers, insectes, animaux marins autres que les poissons...), les verbes qui expriment leur mode d'existence, comme "pulluler", "grouiller", n'impliquent aucun dégoût ou autre sentiment négatif mais traduisent au contraire la bénédiction divine que constitue leur extrême fécondité. Cela conduit Mary Douglas à réexaminer la portée effective des tabous à leur égard - ceux-ci concernant l'alimentation, mais aussi le contact avec les cadavres et, par suite (en principe), l'usage du cuir, de la fourrure, etc. Or les listes cumulées des animaux impurs et abominables reviennent à rendre indisponibles la plupart des espèces : il y a donc là un effet objectif de protection qui témoignerait de l'amour de Dieu pour toutes ses créatures. La disqualification apparente, par une sorte de ruse de la raison, serait en réalité la marque d'une attention bienveillante.

Cette lecture comporte assurément des éléments convaincants. En tout état de cause, je n'ai pas les compétences pour évaluer la pertinence des données linguistiques ou historiques qui la soutiennent. Le principal problème qu'elle pose est que, de l'avis même de l'auteure, elle met au jour une doctrine du Lévitique qui est restée sans effet : le judaïsme l'a toujours assimilée à celle du Deutéronome à laquelle, me semble-t-il, elle ressemble beaucoup plus que ne veut bien l'admettre Mary Douglas. Or le souci de privilégier les singularités du Lévitique la conduit à passer sous silence le cas des animaux qui, comme le porc, sont à la fois abominables et impurs, ou encore à n'aborder la classification des oiseaux qu'à travers l'exemple de la caille. Quant à la thèse de l'amour égal de Dieu pour toutes ses créatures (attestée par ailleurs dans la Bible hébraïque), elle n'annule pas celle d'une hiérarchie de la création dont il faut aussi comprendre le sens. 
Elle a cependant le mérite d'inviter à un réexamen des nombreux textes qui, dans le Lévitique et ailleurs, proscrivent la cruauté envers les animaux ou leur exploitation abusive et invitent à les traiter avec mansuétude. De telles prescriptions traduisent sans doute une orientation éthique particulière, mais suffisent-elles à spécifier une forme originale de pensée de l'animal ? En contexte "naturaliste", on ne disait guère autre chose dans les cours de morale destinés aux enfants de l'école laïque que j'ai connue dans les années 1950. La Bible hébraïque est heureusement assez riche en références aux animaux pour offrir un dossier plus significatif.

Au total, mon analyse prendra en compte certains résultats du livre de Mary Douglas en négligeant, pour l'essentiel, la possible singularité du Lévitique. Sa cohérence avec les autres textes que j’invoquerai ne soulève à mon sens aucune difficulté. Quant au fond, mon sentiment est que les questions soulevées par Mary Douglas gagnent à être replacées dans le contexte théorique plus large que propose l'ouvrage de Philippe Descola.

L'objectif de Par-delà nature et culture ne se réduit pas, bien loin de là, à une étude du statut de l'animal dans différents espaces culturels. Mais, parmi les non-humains dont Philippe Descola analyse les représentations et les relations avec le monde des hommes, l'animal est sans doute celui qui manifeste le mieux les contrastes entre les quatre types d'ontologie auxquels sont ramenées toutes les cosmologies non seulement découvertes empiriquement dans le monde, mais logiquement possibles. Notre expérience immédiate d'êtres à la fois incarnés, vivants et conscients ne peut en effet manquer de rencontrer la question que pose à notre humanité la présence d'êtres proches, à bien des égards, de la représentation que nous avons de nous-mêmes. L'extension de ce registre de la proximité aux plantes ou à la nature inorganique est également attestée (surtout pour les vivants), tout comme une extension beaucoup plus commune concernant les entités surnaturelles - dieux, esprits ou ancêtres -, mais l'animal reste le meilleur candidat à ces évaluations comparatives. D'où mon choix de réduire la mise en œuvre des hypothèses de Descola à la seule confrontation des statuts de l'homme et de l'animal: même si dans certains cas la prise en compte des non-humains est plus large, c'est la question de l'animal qui me semble susciter les constructions les plus significatives.

Or les ressemblances et les différences entre l'homme et l'animal, également prégnantes, se ramènent à une combinatoire fondée sur les deux grandes catégories de l'expérience que nous pouvons avoir de nousmêmes : nous sommes des êtres corporels dotés d'une conscience et d'une intentionnalité - dans le langage de Descola, d'une physicalité et d'une intériorité. La physicalité évidente des animaux s'accompagne, à travers 
les connaissances que nous avons de leurs comportements, capacités cognitives ou productives, etc., d'inférences spontanées de la présence en eux d'une "intériorité » comparable à la nôtre. Sur les deux plans, tout est affaire ensuite d'évaluation: on peut privilégier les continuités ou les discontinuités physiques (nous partageons avec les animaux les grandes fonctions biologiques de l'alimentation, la reproduction etc., mais nous nous distinguons aussi de beaucoup d'entre eux par les modes de locomotion, les ressources alimentaires, etc.). Cela vaut aussi pour les intériorités et pour leurs expressions sensibles: jusqu'à quel point des compétences communes, mais aussi des écarts dans leur mise en œuvre, sont-ils l'indice de proximités ou de différences? En l'absence de toute instance transcendante apte à trancher objectivement de ces questions, on ne peut que s'en remettre à des décisions interprétatives qui se ramènent logiquement à quatre et correspondent à autant d'hypothèses ontologiques relatives à l'homme et à l'animal. Je reprends ci-dessous le tableau des quatre ontologies tel que l'établit Philippe Descola (2005: 176) :

\begin{tabular}{|c|c|c|c|}
\hline $\begin{array}{l}\text { ressemblance des } \\
\text { intériorités } \\
\text { différence des } \\
\text { physicalités }\end{array}$ & animisme & totémisme & $\begin{array}{l}\text { ressemblance des } \\
\text { intériorités } \\
\text { ressemblance des } \\
\text { physicalités }\end{array}$ \\
\hline $\begin{array}{l}\text { différence des } \\
\text { intériorités } \\
\text { ressemblance des } \\
\text { physicalités }\end{array}$ & naturalisme & analogisme & $\begin{array}{l}\text { différence des } \\
\text { intériorités } \\
\text { différence des } \\
\text { physicalités }\end{array}$ \\
\hline
\end{tabular}

Ce tableau ne suffit pas, bien évidemment, à donner une définition satisfaisante de chacun des schèmes ${ }^{7}$. Le livre en propose des attestations ethnographiques ou historiques qui permettent en même temps de compléter l'inventaire de leurs caractères distinctifs. J'intégrerai au fil de l'analyse ces

7. La pertinence de ce schéma et son adaptation à différents espaces culturels ont été discutées. Voir dans L'Homme, le débat entre Jean-Pierre Digard et Philippe Descola (2006, 177-178 : 413435) ; dans la Revue française de sociologie, les notes critiques de Richard Pottier \& François Héran (2007, 48 (4) : 781-793); dans Études rurales, «Autour du livre de Philippe Descola ", de Emmanuel Lézy \& Gérard Chouquer (2006, 178 : 231-252). Même si je partage certaines des interrogations de ces auteurs, je n'ai pas jugé nécessaire d'en faire état ici, mon parti pris étant de tenir pour acquises les hypothèses de Descola et de mettre à l'épreuve leur valeur heuristique. 
données, sans lesquelles des « reconnaissances " hâtives n'auraient guère de pertinence et pourraient même induire en erreur. Mais cette forme simplifiée rend déjà possible une relecture des textes bibliques qui fait vaciller quelques certitudes généralement assumées sans véritable examen.

\section{Les animaux dans l'ordre cosmique}

Les trois premiers chapitres de Genèse offrent une cosmogonie suivie d'une anthropogonie particulièrement resserrées : par comparaison, la Petite Cosmogonie portative de Raymond Queneau fait figure d'encyclopédie ${ }^{8}$ ! Cette brièveté ne facilite pas le travail de l'anthropologue. Les biblistes, quant à eux, ont depuis longtemps repéré la couture approximative d'un premier chapitre «sacerdotal», d'origine post-exilique, avec les chapitres deux et trois, qui reprennent des traditions plus anciennes et sont marqués par l'influence des mythes babyloniens (Gibert 1986). Et de l'un aux autres, en effet, la représentation des animaux varie de façon assez nette.

\section{La construction du cosmos (Genèse I)}

Les six jours de la création renvoient à trois types d'actes créateurs :

- Des ordres directement suivis de la venue à l'être des réalités concernées ( $1^{\mathrm{er}}, 2^{\mathrm{e}}, 3^{\mathrm{e}}$ et $4^{\mathrm{e}}$ jours). Ces actes s'accompagnent d'opérations de séparation (lumière/ténèbres, eau d'en haut/eau d'en bas, eau d'en bas/terre, jour/nuit) qui conferent aux objets visés leur fonction propre. Ils concernent des réalités inorganiques, ou si l'on veut les cadres cosmiques de l'existence.

- Des ordres donnés à un élément de produire à son tour, soit des actes de création indirecte : la terre est invitée à produire des plantes ( $3^{\mathrm{e}}$ jour), puis l'eau les êtres aquatiques ( $5^{\mathrm{e}}$ jour), la terre enfin des animaux classés en bestiaux, bestioles et bêtes sauvages ${ }^{9}$ ( $\sigma^{\mathrm{e}}$ jour). Le cas des oiseaux est plus indécis. Ils sont, semble-t-il, engendrés par l'air, troisième élément non mentionné explicitement. Au principe de séparation de la rubrique précédente correspond ici un principe de spéciation: les plantes portent semence, les animaux apparaissent classés en espèces et doivent en outre se reproduire «selon leur espèce" (animaux terrestres).

8. La palme de la concision revient cependant à l'anthropogonie de Queneau : «Le singe (ou son cousin) le singe devint l'homme/ lequel un peu plus tard désagrégea l'atome "...

9. Les plantes, distinguées en herbes portant semence et en arbres portant des fruits, bénéficient elles aussi d'un service taxinomique minimum, correspondant à l'échelle des "formes de vie » identifiées par les ethnosciences, en omettant cependant l'étage intermédiaire des buissons ou arbustes. 
- Un ordre que Dieu se donne à lui-même ${ }^{10}$ et qui participe donc lui aussi des actes de création indirecte, relevant de l'engendrement. Il vaut pour l'homme seul. Le principe d'individuation ne peut plus être ici la séparation ou la spéciation puisqu'une seule catégorie d'êtres est concernée. Son équivalent semble être la ressemblance qui s'instaure entre créateur et créature - un principe de similarité qui n'est du reste pas absent des autres actes de création indirecte : chaque catégorie de vivants est associée de façon au moins métonymique à l'élément qui lui a donné naissance.

Mis à part le quatrième jour (création des luminaires célestes) qui vient bizarrement s'intercaler entre la création des plantes et celle des animaux, l'ordre temporel des actes créateurs répond à un principe hiérarchique: on passe de l'inorganique au vivant, puis à l'humain. Cette hiérarchie est explicitée en Gn 1, 28-30: il revient à l'homme de dominer sur les animaux et de consommer les plantes. À noter aussi la distinction entre l'inorganique et le vivant (humain compris), toujours issu d'actes de création indirecte. Un principe d'engendrement, souvent exprimé dans les termes de la multiplication, du grouillement, apparaît ainsi comme caractéristique de la vie. Cette première disjonction s'accompagne cependant d'une seconde, à l'intérieur du vivant: l'homme relève d'un principe d'engendrement distinct de celui des animaux, même s'il est ensuite soumis comme eux à la reproduction, ou à un principe de fécondité.

Une autre classification, plus subtile, vient enrichir la précédente. Elle concerne cette fois-ci les groupes d'êtres créés le même jour : la terre ferme et les plantes le $3^{\text {e }}$, les êtres aquatiques et les oiseaux le $5^{\mathrm{e}}$, les animaux terrestres et l'homme le $6^{\mathrm{e}}$. Cela suggère une similitude de position des poissons et des oiseaux par rapport à l'homme : ils sont plus éloignés de lui

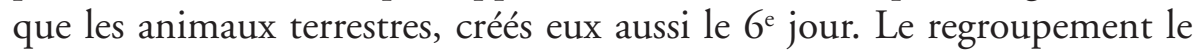
plus surprenant est celui du $3^{\mathrm{e}}$ jour qui, en associant l'émergence de la terre ferme et la naissance des plantes, semble rapprocher ces dernières de l'inorganique et en faire des éléments du "décor " plutôt que de véritables acteurs sur le théâtre du monde. Si tel est bien le cas, on comprend mieux le report au $4^{\mathrm{e}}$ jour de la création des luminaires célestes : les fonctions qui leur sont reconnues - éclairer la terre avec plus ou moins d'intensité et rendre possible, grâce à la distinction des jours, la construction du calendrier - relèvent elles aussi de l'ordonnance du décor. En outre, le contraste nuit/jour est pertinent pour la vie animale comme pour la vie humaine

10. D'où peut-être le passage au pluriel (Faisons l'homme à notre image) qui a fait couler tellement d'encre: le dieu sujet des actes de création précédents s'adresse ici à lui-même en tant qu'objet, principe d'engendrement de l'homme. 
mais n'affecte pas les plantes (sauf exceptions) de manière visible. Son institution à ce moment-là parachève donc la mise en place des cadres cosmiques requis par l'existence des formes supérieures de la vie.

Essayons à présent de confronter ces données aux catégories proposées par Philippe Descola. S'agissant du rapport des hommes aux nonhumains, on voit apparaître une double discontinuité : du point de vue des physicalités, l'homme se distingue des animaux par l'opposition espèce unique/espèces plurielles et surtout par son principe d'engendrement, non lié à un élément (terre, eau) et fondé sur une encore énigmatique "ressemblance" avec Dieu. N'est-ce pas là, précisément, le lieu d'affirmation d'une singularité des hommes quant aux «intériorités "? Du moins, la ressemblance à Dieu est difficilement pensable sur le plan physique et le Dieu qui a été mis en scène semble doté d'une intériorité analogue à celle des hommes. Cette hypothèse d'une double distinction des physicalités et des intériorités entre les hommes et les animaux sera approfondie à la lumière d'autres textes. Il semble en tout cas que ce trait essentiel du modèle analogique fasse écho au climat global de ce premier chapitre. On peut en effet considérer les six jours de la création comme un tableau manifestant à la fois la mise en correspondance de séries (les oiseaux sont à l'air ce que les poissons sont à l'eau et les animaux à la terre; la séparation est aux entités cosmiques ce que la spéciation est aux vivants et la ressemblance à Dieu aux hommes, etc.) et un principe hiérarchique : deux traits caractéristiques, selon Descola, de l'ontologie analogiste.

\section{Où l'animisme pointe son nez (Gn 2-3)}

Le second récit de création de $\mathrm{Gn} 2$, considéré comme plus archaïque, modifie sensiblement l'ordre, la distribution et la nature des actes créateurs. Par voie de conséquence, les lignes de partage entre catégories de créatures humaines et non humaines se trouvent redessinées. Cette fois, la création de l'homme est première. Alors que la terre était encore déserte et seulement humectée par de l'eau d'en bas, "Yahvé Dieu modela l'homme avec la glaise du sol, il insuffla dans ses narines une haleine de vie et l'homme devint un être vivant» $(2,7)$. La création des plantes, qui vient ensuite, relève du même procédé de création indirecte que précédemment: Dieu les fait "pousser du sol» $(2,9)$. Quant aux animaux, destinés à devenir les compagnons d'un homme encore solitaire, ils sont comme lui «modelés du sol» $(2,19)$ et, on peut le supposer, animés de la même manière. Mais les animaux s'avèrent des compagnons décevants. D'où la création de la femme, par bouturage, celle-ci étant reconnue semblable matériellement à l'homme originel : "À ce coup, c'est l'os de mes os et la chair de ma chair! " $(2,23)$. En quoi ces premiers humains se différencient-ils des animaux? 
Comme eux, ils sont nus et n'en ont pas honte. Rien n'indique qu'ils soient voués à la reproduction sexuelle mais, à cet égard, on n'en sait pas davantage à propos des trois classes d'animaux mentionnées (bestiaux, oiseaux et bêtes sauvages). En fait, cette création ayant pour cadre le seul jardin d'Éden, et non la terre sans autre spécification, il semblerait que les animaux soient des prototypes dont la seule pluralité valorisée est celle des espèces (ce mode de distinction se retrouvant dans l'épisode de la nomination $[2,19-20])$. Au total, ce passage pose avec une assez grande netteté le principe d'une différenciation des physicalités qui concerne les animaux les uns par rapport aux autres et l'homme par rapport à eux, tous étant issus d'une suite d'opérations distinctes de modelage. Bénéficiant d'un même type d'animation ${ }^{11}$, ils ne peuvent se distinguer que par la forme de leur corps. On comprend, dans cette logique, que la femme ne puisse être modelée à partir de la terre : cela reviendrait à suggérer qu'elle est, comme les animaux, d'une autre espèce que l'homme.

Qu'en est-il à présent des intériorités? La différence entre la vie et l'inorganique s'exprime ici par la présence du souffle divin, celui-ci instituant en même temps entre l'homme et les animaux une continuité qui me semble se situer du côté des intériorités. En effet, en dehors de toute autre indication (comme tout à l'heure la ressemblance avec Dieu), il est acquis 1) que l'homme parle 2) que les animaux peuvent être considérés comme des " compagnons " acceptables - et donc doués de la parole ? - du premier humain. Cette propriété commune, si elle existe, ne peut résulter que de ce qu'ils ont en commun et qui les distingue des plantes et de l'inorganique, à savoir le souffle divin. Or le fait que les animaux édéniques possèdent la parole est confirmé par l'épisode de la chute: la femme n'est nullement surprise lorsque le serpent s'adresse à elle... Un serpent qualifié, du reste, de "plus rusé de tous les animaux» $(3,1)$, ce qui suppose que ce trait bien humain d'intériorité est partagé à des degrés divers par les autres espèces. Enfin le serpent (dont rien dans le texte ne suggère qu'il est une incarnation du démon) est puni - puni en tant qu'animal : un acte qui présuppose une intériorité de type humain.

Discontinuité des physicalités et continuité des intériorités : nous voici en présence des deux traits définitionnels du modèle animiste. Ce changement de modèle par rapport au premier chapitre est troublant, mais nous savons qu'il s'agit de textes d'âge et d'inspiration différents. Je remets à plus tard la discussion de ce résultat d'autant plus dérangeant que, comme on va le voir, il peut être étayé par des références scripturaires plus explicites.

11. On peut se demander si l'épisode de la nomination des animaux par l'homme n'est pas l'équivalent de l'insufflation par Dieu de la vie au premier homme. Si tel est le cas, il y a là un indice de l'activité du modèle analogique (Dieu est à l'homme ce que l'homme est aux animaux). 


\section{Les animaux dans l'Alliance (Gn 6-9)}

92 D'animaux, il n'est guère plus question dans les chapitres 4 et 5 de Genèse, qui décrivent la vie des premiers humains chassés du paradis. La seule mention est celle du sacrifice par Abel des premiers-nés de son troupeau $(4,3)$. Les animaux reviennent en force avec l'épisode du déluge. On ne s'étonne pas assez du fait qu'ils sont associés à un châtiment qui, a priori, concerne uniquement les hommes. En fait, cet épisode va nous apporter des indications précieuses sur la place de chacun dans l'ordre du monde et sur l'ontologie sous-jacente à ce classement.

\section{Punition et Alliance}

Pourquoi le déluge, qui doit-il concerner? Au début du chapitre 6, Yahvé fait état de ses griefs : les «fils de Dieu " prennent pour femmes les "filles des hommes». On a beaucoup glosé sur l'identité de ces nouveaux venus dont les accouplements excitent la colère divine. Peu importe pour mon propos. L'important est que cela déclenche un premier acte punitif : "Que mon esprit ne soit pas indéfiniment humilié dans l'homme, puisqu'il est chair; sa vie ne sera que de cent vingt ans» $(6,3)$. Nous apprenons par la même occasion qu'une part de «l'esprit de Dieu » est présente dans les hommes et que cette cohabitation doit respecter un certain équilibre : va pour la chair, mais point trop n'en faut! Aussi l'excès de chair est-il deux fois corrigé : par le raccourcissement de la vie humaine (jusqu'alors, les hommes vivaient 700 ou 800 ans : voir Gn 5); par l'élimination, dans les eaux du déluge, des Néphilim, en qui la tradition reconnaît des géants, autrement dit des êtres marqués eux aussi par un excès de chair.

Ce n'est pas tout. La méchanceté des hommes déborde, Yahvé se repent de les avoir créés, puis il ajoute: "Je vais effacer de la surface du sol les hommes que j'ai créés - et avec les hommes les bestiaux, les bestioles et les oiseaux du ciel - car je me repens de les avoir faits » $(6,7)$. On ne voit pas très bien de quoi les animaux se sont rendus coupables, mais ils n'en sont pas moins les cibles explicites de la colère divine. Cependant, de même que Dieu trouve en Noé le seul homme digne d'être sauvé et de donner naissance à une nouvelle humanité, de même il exempte chaque espèce animale du désastre et lui donne un futur, tout en annihilant la plupart de ses représentants actuels. Pour l'heure, c'est «toute chair» qui doit être détruite. C'est alors qu'apparaît pour la première fois dans le texte une distinction d'un nouveau genre entre les animaux : certains sont purs, d'autres impurs (7, 2-3). Une distinction qui s'accompagne, semble-t-il, d'une hiérarchie : sept couples des premiers seront sauvés, un seul des seconds. 
Les éléments les plus importants pour notre enquête interviennent toutefois dans l'épisode qui suit le déluge, celui de la première Alliance entre Yahvé et « toute chair ». La résolution divine de maintenir désormais l'ordre cosmique a pour contrepartie le respect de certaines règles par les hommes, et aussi par les animaux, si l'on prend le texte à la lettre, pour au moins l'une d'entre elles. Je dois citer dans son intégralité ce passage essentiel, où Yahvé s'adresse à Noé et à ses fils $(9,2-7)$ :

"Soyez féconds, multipliez, emplissez la terre. Soyez la crainte et l'effroi de tous les animaux de la terre et de tous les oiseaux du ciel, comme de tout ce dont la terre fourmille et de tous les poissons de la mer: ils sont livrés entre vos mains. Tout ce qui se meut et possède la vie vous servira de nourriture, je vous donne tout cela au même titre que la verdure des plantes. Seulement, vous ne mangerez pas la chair avec son âme, c'est-à-dire le sang. Mais je demanderai compte du sang à chacun de vous. J'en demanderai compte à tous les animaux et à l'homme, aux hommes entre eux, je demanderai compte de l'âme de l'homme.

Qui verse le sang de l'homme,

Par l'homme aura son sang versé.

Car à l'image de Dieu

L'homme a été fait.

Pour vous, soyez féconds, multipliez, pullulez sur la terre et la dominez. »

Le principe d'animation commun aux hommes et aux animaux est, pour la première fois dans ce texte, désigné comme ce que j'appellerai l'âmesang: celle-ci ne doit pas être consommée ni, dans le cas de l'homme, épanchée par l'homicide. Le Lévitique précisera qu'elle doit revenir à Dieu lors des sacrifices d'animaux. En même temps, il est désormais établi que l'homme a le droit de se nourrir de chair animale, sous la condition que l'on vient de voir. Cette nouvelle possibilité s'accompagne d'une formulation abrupte de la hiérarchie homme/animal déjà mentionnée en Gn1, 2829. La dignité supérieure des hommes s'exprime aussi à travers la prohibition de l'homicide, motivée par le fait que l'homme a été fait "à l'image de Dieu». Enfin, l'intégration des animaux à l'Alliance est explicitement conditionnée par la non-consommation de sang, ce qui revient de fait à en exclure les carnivores. Un troisième registre de législation associé à cet épisode peut être inféré de la mésaventure de Cham qui, découvrant son père dénudé, s'en moque au lieu de le couvrir de son manteau, et qui est maudit par Noé. L'épisode est obscur, mais on s'accorde à y reconnaître une allusion aux règles régissant la sexualité ${ }^{12}$, qui trouveront dans le Lévitique seulement leur pleine expression.

12. Le principal argument est que la formule "voir la nudité de ", pertinente au sens littéral dans l'épisode de Cham, est utilisée dans le Lévitique pour désigner un rapport sexuel. Edmund Leach (1980) n’hésite pas à conclure à une relation homosexuelle entre Cham et son père. 
Essayons à présent de rapprocher ces nouvelles données de ce que nous savions déjà de la nature et du statut respectif des vivants. Comme je viens de le faire à deux reprises, cette nouvelle étape de l'analyse me conduira désormais à faire des références fréquentes aux textes du Lévitique, source par excellence sur les questions dont nous allons traiter : pureté, alimentation, sexualité, sacrifice.

\section{Hiérarchies et devoirs}

Les éléments nouveaux que nous venons d'intégrer en suivant le fil du texte biblique confirment sur l'essentiel ce que nous savions déjà, moyennant peut-être la mise en équivalence de notions qui semblent jouer un rôle similaire :

- l'animation due au souffle divin (et à celui d'Adam ?) est désormais attribuée à l'âme-sang;

- la ressemblance avec Dieu peut être rapprochée de la notion d' « esprit » de Dieu, l'un et l'autre étant caractéristiques de l'homme et de lui seul.

Nous retrouvons aussi la dualité entre un principe hiérarchique fondé sur la différence des intériorités et une proximité peut-être encore renforcée à cet égard entre l'homme et l'animal du fait de leur commune participation à l'Alliance. En revanche, le monde animal s'est enrichi d'une distinction nouvelle, celle des animaux purs et impurs. Mais celleci, à la différence de ce qui sera mis en place dans le Lévitique, n’a pas d'incidence sur l'alimentation. Il est précisé que Noé ne sacrifie que des animaux purs, mais rien ne permet d'affirmer que seules des bêtes sacrifiées sont consommées. Je résume dans le tableau ci-dessous les relations existant, à ce point du texte, entre disponibilité alimentaire et principes d'animations ou intériorités :

\begin{tabular}{|c|c|c|}
\hline Types d'êtres & Alimentation & $\begin{array}{c}\text { Modes d'animation, } \\
\text { types d'intériorités }\end{array}$ \\
\hline Plantes & $\begin{array}{c}\text { Nourriture des } \\
\text { animaux et de l'homme }\end{array}$ & Pas d'âme-sang \\
\hline Animaux & $\begin{array}{c}\text { Nourriture des } \\
\text { animaux et de l'homme }\end{array}$ & Âme-sang \\
\hline Homme & Non-nourriture & $\begin{array}{c}\text { Âme-sang }+ \\
\text { ressemblance avec Dieu } \\
(=\text { esprit?) }\end{array}$ \\
\hline
\end{tabular}


La question la plus embarrassante est celle du sens à donner à la participation des animaux à l'Alliance. Cette dernière se présente en effet comme un engagement réciproque qui, dans le cas des hommes au moins, suppose l'obéissance à des lois et, par conséquent, une intériorité de type humain. La question s'était déjà posée à propos du déluge : pour mériter d'être punis, les animaux devaient avoir péché, au sens humain du terme. De quoi s'étaient-ils donc rendus coupables? Cette question - faut-il s'en étonner ? - a déjà été envisagée par d'anciens commentateurs juifs, qui me semblent lui avoir apporté une réponse en parfait accord avec l'esprit, sinon la lettre, du texte biblique: la faute des animaux est qu'ils se sont hybridés, tout comme le faisaient à leur manière les "fils de Dieu » et les «filles des hommes ». Or se reproduire "selon son espèce" est un commandement (ou une norme de la création) qui revient quatre fois dans Gn1, l'hybridation volontaire des bestiaux étant quant à elle prohibée par le Lévitique : "Tu n'accoupleras pas dans ton bétail deux bêtes d'espèce différente" $(19,19)$. Hélas pour eux, avant le déluge, les manquements des animaux à cette règle furent nombreux. Si l'on en croit un ancien talmudiste, « les bêtes elles-mêmes repoussaient leurs partenaires propres : l'étalon montait l'ânesse; le baudet, la jument; le chien, la louve; le serpent, la tortue, et ainsi de suite» (Graves \& Patai 1987 : 122).

La pratique de l'hybridation est ici clairement rapportée à une perversion du monde animal lui-même, comme le suggère du reste Gn6, 12 : " toute chair avait une conduite perverse sur la terre». On voit donc se confirmer une première lecture possible du sens à donner à la participation des animaux à l'Alliance : considérés comme dotés d'une intériorité de type humain, ils peuvent eux aussi se comporter ou non de façon méritoire. Cette conception "animiste" de l'animal a eu sa propre descendance, au moins dans le christianisme : le Moyen Âge a connu des procès d'animaux coupables d'homicides. Je reviendrai plus loin sur cette question. Cela étant, on imagine mal, comme je le notais plus haut, qu'un carnivore puisse s'alimenter en séparant la chair du sang. Il y aurait donc des animaux qui, par nature, seraient exclus de l'Alliance : une hypothèse qui, soit dit en passant, contredit l'idée, avancée par Mary Douglas, d'un Dieu aimant également toutes ses créatures.

En vérité, l'examen du cas des animaux carnassiers nous situe déjà dans une perspective différente de celle que je viens de qualifier d'animiste : les animaux ont-ils vraiment le choix d'entrer dans l'Alliance ou d'en sortir? La vie animale ne se situe-t-elle pas dans une autre relation à cette alternative? Pour clarifier ce point, je propose de comparer ce mode de classement des animaux, seulement suggéré par les textes, avec un autre qui, lui, est tout à fait explicite : leur partition en purs et impurs. Or, si l'on suit 
le Lévitique, on remarque que tous les carnivores et les charognards (animaux terrestres ou oiseaux) sont dits impurs ou abominables, et par conséquent interdits de consommation. On peut de la même manière mettre le péché d'hybridation (versant des règles de l'Alliance relatives à la sexualité) en relation avec l'état d'hybridité par nature qui est celui des animaux, tels le porc ou le daman, classés impurs en raison de la co-présence en eux de caractères typiques d'espèces différentes ${ }^{13}$. Le cas de certains animaux marins relève peut-être de la même logique : les crustacés seraient des hybrides d'êtres terrestres et marins, les coquillages uniraient animal et minéral. Quant aux bestioles abominables terrestres, je suggère qu'elles pèchent par un défaut de spéciation et/ou de distinction d'avec l'élément qui leur donne naissance: soit que l'on explique leur venue à l'être par quelque variante d'une théorie de la génération spontanée, soit que, rampantes ou souterraines, elles n'aient pas gagné l'élévation au-dessus du sol (la séparation d'avec la terre) qui, selon des traditions talmudiques, en ferait des candidates légitimes à la pureté, comme la bondissante sauterelle.

Il y aurait donc un isomorphisme, chez les animaux, entre non-respect des lois de l'Alliance et impureté ou "abomination " ${ }^{14}$, celle-ci étant rapportée à des caractères naturels des espèces. Cette manière d'envisager les choses fait l'économie d'une intériorité animale de type humain. À ce point de l'analyse, il semble plutôt que, dans l'ordre de la création, une analogie existe entre l'homme et l'animal, également qualifiables par rapport à la catégorie de pureté mais distincts dans leur manière d'être ou de ne pas être en accord avec les règles qui la procurent : cela résultant d'un acte de volonté pour les premiers et d'une nature conforme ou non conforme pour les seconds. Nous verrons qu'en réalité cette opposition entre volonté et nature doit être nuancée pour ce qui concerne les hommes. Du moins les animaux incarnent-ils la conformité ou la transgression, la vertu ou le vice : s'il est permis ici encore d'anticiper, c'est exactement ainsi qu'ils apparaissent dans les bestiaires chrétiens.

Cette seconde lecture suggère donc un basculement du côté de l'analogisme. Mais elle reste insuffisante pour deux raisons au moins :

- D'une part, en assimilant appartenance à l'Alliance et pureté, j'ai brûlé les étapes. En effet, comme le souligne à juste titre Mary Douglas, les règles de pureté valent pour les seuls Hébreux, alors que le contrat

13. On notera que les hybrides connus d'expérience (le mulet, peut-être l'ovicapre) ou imaginés (comme le léopard au Moyen Âge) réunissent des traits issus de leurs deux espèces d'origine, ce qui les rapproche du cas des «hybrides par nature ».

14. Une hypothèse à valider : l'impureté serait liée à l'hybridation objective, l'abomination au régime alimentaire ou à d'autres anomalies de moindre importance. 
noachique vaut pour "toute chair». Il reste donc à spécifier les relations entre pureté et Alliance et, pour cela, à définir la position singulière des Hébreux dans l'ordre de la création.

- D'autre part, si l'on suit le modèle de Philippe Descola, une ontologie analogiste construit des séries à la fois continues et discontinues d'existants au lieu de se réduire aux couples superposables d'opposition homme/animal et nature/culture typiques du naturalisme. La reconnaissance d'une ontologie analogiste dans ces textes gagnerait donc beaucoup à la mise en évidence de séries de ce genre, par-delà les distinctions encore assez pauvres que nous avons pu noter entre les animaux (purs/impurs) et entre les hommes (intégrés ou non à l'Alliance avec Noé). De plus, l'analogisme n'est pas seulement un système classificatoire statique, il est avant tout une matrice permettant de penser des formes de circulation entre différentes catégories d'êtres d'abord identifiés par leurs ressemblances et leurs différences, mais aussi porteurs d'une pluralité de supports d'identité (âmes, double, ombre...) qui donnent à chacun son individualité du fait d'une composition unique. Or seuls les aspects classificatoires ont, jusqu'ici, été repérés. Qu'en est-il donc des transactions, médiations, assimilations entre les différentes échelles d'êtres? En quoi les propriétés substantielles qu'ils partagent sont-elles susceptibles de circuler entre eux?

\section{De l'ordre cosmique à la sainteté}

"Soyez saints parce que je suis saint.» Cette injonction divine, qui revient à plusieurs reprises dans le Lévitique, a été à bon droit mise en valeur par Mary Douglas dans De la souillure et, malgré les palinodies de son dernier ouvrage, elle continue d'y souligner le fait que cette demande s'adresse uniquement aux Hébreux : eux seuls en effet sont soumis aux lois de pureté et aux autres prescriptions énumérées par le Lévitique, c'està-dire par le texte qui développe le cadre mosaïque de l'Alliance. Cette remarque invite à préciser les relations entre la diversification des peuples, thème essentiel tout au long de Genèse, et les obligations liées à l'Alliance. Ce sera aussi une occasion d'y voir plus clair sur la nature des systèmes de différences envisagés jusqu'ici entre les créatures.

\section{Unité de l'espèce humaine et diversité des peuples}

Plus encore que l'animisme et le totémisme, l'analogisme selon Philippe Descola donne une dimension cosmique aux composants et aux qualités essentielles de la personne humaine. Par exemple, les catégories du chaud et du froid valent pour classer les tempéraments, les maladies, mais aussi les plantes, les minéraux, etc. L'anthropogenèse biblique semble, a priori, 
n'accorder aucune place à ce type de catégorisations abstraites, elle se rapprocherait plutôt des systèmes "à composants multiples ", comme ceux des Dogons ou des anciens Mexicains (Descola 2005 : 288-313), même si elle est beaucoup plus économe en principes constituants (corps, âme sang, esprit...) et plus explicitement hiérarchique : ces principes construisent un ordre significatif des créatures selon une logique présence/absence plus que par la modulation, quantitative ou autre, des effets en chaque type d'êtres de réalités substantielles en même temps diffuses dans l'ensemble du cosmos. Mais on peut se demander si, après le classement hiérarchique des animaux, la distribution de l'humanité en différents peuples ne met pas en jeu des principes évoquant une théorie (elle aussi discriminante et hiérarchique) des composants de la personne.

Un constat, pour commencer : la transgression apparaît, dans Genèse, comme la formule la plus fréquente de l'ethnogenèse. Déjà l'homicide de Caïn crée la lignée des Caïnites (détruits dans le déluge ?), par différence avec celle des fils de Seth. La transgression de Cham (et sa malédiction) est à l'origine des Cananéens et d'autres peuples voués à la servitude. Celle de Lot (relation incestueuse avec ses filles) engendre les Moabites et les Ammonites. Le comportement d'Agar à l'égard de Sara conduit à la séparation des Ismaélites. On peut enfin supposer que les autres peuples évoqués dans les textes (Égyptiens, Hittites, etc.) sont eux aussi issus d'ancêtres non mentionnés situables à un niveau ou un autre des bifurcations explicites.

Il ne saurait y avoir de transgression en l'absence de règles. Or une des convictions qui semble inspirer ces textes avec le plus de constance est que l'existence de l'humanité est inséparable de la position d'une Loi. Tel est évidemment le principe même de l'Alliance, mais il se trouve déjà à l'œuvre dans le jardin d'Éden: le projet d'humanité envisagé par Dieu pour les premiers hommes était déjà lié au tabou sur les fruits de l'arbre de la connaissance. Aussi sa violation entraîne-t-elle une première bifurcation dans l'histoire de l'humanité : l'homme pécheur, devenu mortel et voué à la reproduction sexuelle, au travail (etc.), diverge de l'humanité (seulement virtuelle) ${ }^{15}$ qui serait née d'un Adam innocent. La faute ouvre du reste une forme de similarité entre les hommes et Dieu que je n'avais pas notée jusqu'ici. Yahvé dit en effet $(\mathrm{Gn} 3,22)$ : "Voilà que l'homme est devenu comme l'un de nous, pour connaître le bien et le mal! "Est-ce là un équivalent de la relation de "ressemblance» mentionnée en Gn1 ? Peut-être. En tout cas il s'agit, comme beaucoup de commentateurs (dont Kant) l'ont notée, de l'acquisition par l'homme d'une capacité qui doit être

15. C'est elle, selon la très convaincante analyse de Hans Belting, qui est figurée sur le panneau central du Jardin des délices de Jérôme Bosch (Belting 2005). 
considérée comme une composante essentielle de son intériorité (et de celle de Dieu). Peu importe qu'on l'appelle libre arbitre, conscience morale ou disposition à reconnaître une loi : l'important est le découplage qui en résulte entre un en-soi, un pur donné et un devoir-être constitutif de la réalité humaine elle-même.

On a tiré déjà bien des conséquences théologiques et philosophiques des idées dont je me fais ici l'écho. Mon projet, beaucoup plus modeste, sera d'examiner ce qui en résulte dans la perspective des anthropogenèse et ethnogenèse bibliques. Mon hypothèse est que l'obéissance à un type de loi divine ou à un stade de l'Alliance peut être traitée comme une propriété ontologique d'un type particulier (éventuellement cumulable avec d'autres) qui caractérise d'abord l'appartenance à un peuple, puis la "situation ontologique" de chaque homme en particulier. Suivons pour commencer les grandes étapes de l'introduction de la loi et leurs conséquences sur la division des peuples et sur leurs caractères propres.

Passé l'âge édénique, tout commence à vrai dire par une situation d'absence de loi qui dure jusqu'au déluge. Cela explique peut-être les désordres de cette période, voire l'humanité incertaine de quelques-uns des acteurs ("fils de Dieu», géants...). Mais il faut aussi introduire un principe normatif implicite, dont nous verrons bientôt l'importance dans la définition des peuples et des hommes: l'idée que la reconnaissance de devoirs à l'égard de Dieu - une sorte de stade immanent de la loi - persiste à travers une transmission généalogique sélective indexée sur l'aînesse ${ }^{16}$. Celle-ci semble comporter à la fois des devoirs spécifiques envers Dieu (qui iront dans l'Alliance mosaïque jusqu'à l'idée d'une appartenance, ou d'une vocation à Dieu des premiers nés mâles des hommes et des troupeaux) et, en même temps, les ressources pour y faire face. Noé, un aîné descendant d'une lignée d'aînés dont il est dit " qu’il marchait avec Dieu » $(\mathrm{Gn} 6,8)$ est typique de ce statut. La généalogie et l'aînesse apparaissent ainsi comme des composantes de l'identité, individuelle ou ethnique, des personnes : les descendants demeurent dans le rapport à la loi de leur ancêtre. Le jeu de ces deux principes est encore compliqué par l'éventuelle mixité des alliances entre des conjoints issus de peuples différents : une situation qui, comme le notait Edmund Leach (1980), module d'une manière presque toujours jugée sévèrement la stricte appartenance à une lignée élue. Telle serait donc l'une des figures concrètes de l'ontologisation des rapports à la loi et de la hiérarchie qui en résulte. À noter que ce processus est analogue à l'incorporation des mérites que nous avons notée à propos des animaux purs et impurs.

16. À l'exception de Seth, la généalogie des patriarches de Gn5 est celle des aînés de chaque génération. Seth est lui-même une sorte d'aîné, après l'élimination (par la mort ou l'exil) des deux premiers fils d'Adam, auquel les traditions apocryphes attribuent du reste une vaste progéniture. 
Les hommes se distribuent ainsi en fonction de leur conformité aux règles de l'Alliance qui, étape par étape, sont révélées. Un tableau (où je reprends le cas des animaux) résumera cette suite de bifurcations et de positions hiérarchiques et les qualifications qui leur correspondent:

\begin{tabular}{|c|c|c|c|c|c|}
\hline & Lois & Transgresseurs & Transgressions & Sanction & Effet \\
\hline Éden & $\begin{array}{l}\text { Interdit sur le } \\
\text { fruit }\end{array}$ & Adam et Ève & $\begin{array}{c}\text { Consommation } \\
\text { alimentaire }\end{array}$ & Exil, etc. & $\begin{array}{c}\text { Humanité } \\
\text { mortelle }\end{array}$ \\
\hline \multirow{3}{*}{$\begin{array}{l}\text { Âge pré- } \\
\text { diluvien }\end{array}$} & Spéciation & Animaux & Hybridation & - & $\begin{array}{l}\text { Animaux } \\
\text { impurs }\end{array}$ \\
\hline & \multirow[b]{2}{*}{$\begin{array}{l}\text { Justice [valeur } \\
\text { de l'aînesse] }\end{array}$} & Caiin & Homicide & Séparation & Caïnites \\
\hline & & $\begin{array}{l}\text { Hommes } \\
\text { "macrobes" } \\
\text { géants }\end{array}$ & $\begin{array}{l}\text { Unions indues } \\
\text { Violence }\end{array}$ & $\begin{array}{c}\text { Réduction de } \\
\text { la chair } \\
\text { Destruction }\end{array}$ & $\begin{array}{l}\text { Humanité } \\
\text { actuelle }\end{array}$ \\
\hline \multirow{4}{*}{$\begin{array}{c}\text { Contrat } \\
\text { noachique }\end{array}$} & \multirow{2}{*}{$\begin{array}{l}\text { Interdit sur le } \\
\text { sang }\end{array}$} & Animaux & $\begin{array}{l}\text { Régime } \\
\text { carnivore }\end{array}$ & - & $\begin{array}{c}\text { Animaux } \\
\text { abominables }\end{array}$ \\
\hline & & Hommes & $\begin{array}{c}\text { Consommation } \\
\text { du sang }\end{array}$ & Séparation & $\begin{array}{c}\text { Peuples } \\
\text { barbares? }\end{array}$ \\
\hline & $\begin{array}{l}\text { Prohibition } \\
\text { du meurtre }\end{array}$ & Hommes & Homicide & $\begin{array}{l}\text { Châtiment } \\
\text { humain }\end{array}$ & $\begin{array}{l}\text { Hommes } \\
\text { pécheurs }\end{array}$ \\
\hline & $\begin{array}{l}\text { Prohibition } \\
\text { de l'inceste }\end{array}$ & $\begin{array}{c}\text { Cham } \\
\text { Lot et ses filles }\end{array}$ & $\begin{array}{c}\text { Voit la nudité } \\
\text { de Noé } \\
\text { Inceste }\end{array}$ & $\begin{array}{l}\text { Séparation } \\
\text { Séparation }\end{array}$ & $\begin{array}{l}\text { Cananéens } \\
\text { Moabites }^{17} \\
\text { Ammonites }\end{array}$ \\
\hline \multirow{3}{*}{$\begin{array}{c}\text { Alliance avec } \\
\text { Abraham }\end{array}$} & \multirow{3}{*}{$\begin{array}{l}\text { Circoncision } \\
\text { [non mixité } \\
\text { des unions] }\end{array}$} & $\begin{array}{l}\text { Certains } \\
\text { peuples }\end{array}$ & $\begin{array}{c}\text { Non- } \\
\text { circoncision }\end{array}$ & Séparation & "Les Nations" \\
\hline & & Agar & $\begin{array}{c}\text { Irrespect de } \\
\text { Sara }\end{array}$ & $\begin{array}{l}\text { Exil d'Agar et } \\
\text { Ismaël }\end{array}$ & Ismaélites \\
\hline & & Ésaü & $\begin{array}{l}\text { Mépris de } \\
\text { l'aînesse } \\
\text { Mixité }\end{array}$ & Séparation & Édomites \\
\hline \multirow{3}{*}{$\begin{array}{l}\text { Alliance de } \\
\text { Moïse }\end{array}$} & Pureté & $\begin{array}{l}\text { Certains } \\
\text { Hébreux }\end{array}$ & $\begin{array}{l}\text { Pollutions } \\
\text { diverses }\end{array}$ & $\begin{array}{c}\text { Séparation } \\
\text { momentanée }\end{array}$ & \multirow{3}{*}{$\begin{array}{l}\text { Hébreux } \\
\text { pécheurs ou } \\
\text { infidèles }\end{array}$} \\
\hline & Règles morales & Idem & $\begin{array}{l}\text { Injustices } \\
\text { Crimes }\end{array}$ & $\begin{array}{l}\text { Châtiments } \\
\text { humains }\end{array}$ & \\
\hline & Règles du culte & $\begin{array}{c}\text { Hébreux } \\
\text { Lévites } \\
\text { Grand prêtre }\end{array}$ & Sacrilèges & $\begin{array}{l}\text { Châtiments } \\
\text { humains } \\
\text { et divins }\end{array}$ & \\
\hline
\end{tabular}

17. Lépisode de Lot se situe après l'introduction de la circoncision. Rien cependant dans le texte ne permet d'affirmer que Lot (à ce moment-là séparé d'Abraham) a été circoncis. La malédiction de Jérémie (9, 24-25) donne à penser que les Moabites, qui d'ailleurs ne sont pas des descendants directs d'Abraham, sont considérés par les Hébreux comme un peuple incirconcis - ou circoncis seulement «dans la chair». 
Létape décisive est, bien entendu, celle qui sépare les Hébreux des autres peuples et qui marque, à l'issue du cycle d'Abraham, la fin des récits d'ethnogenèse. Seul Ésaü, transgressif au moins par son mépris de l'aînesse ${ }^{18}$, fondera encore la lignée des Édomites consécutive à son union avec une épouse hittite. En revanche, la construction hiérarchique se poursuit (de façon certes équivoque) au sein même du peuple hébreu avec la division en tribus (issues des enfants inégalement légitimes de Jacob-Israël), puis la valorisation, dans Exode et Nombres, de la tribu de Lévi et, en son sein, de la lignée d'Aaron, seule habilitée à fournir les Grands Prêtres.

En résumé, chaque type d'hommes voit son identité définie par le cumul de deux séries de composants : certains innés, relevant de l'en-soi, comme la possession d'une âme-sang, d'un "esprit ", d'une " hérédité " elle-même modulée par le jeu discriminant de l'aînesse et l'éventuelle mixité des unions; d'autres issus d'actes de la volonté comme l'intégrité morale (avec sa branche alimentaire dans le cadre du contrat noachique), la pureté et peut-être la sainteté. La première série conditionne en principe la seconde : par exemple, il faut être un Hébreu pour que les règles de pureté aient du sens, ou encore être issu de la lignée d'Aaron pour pouvoir/devoir accéder à l'état élevé de pureté requis par la fonction de Grand Prêtre. L'intrication des deux séries contribue, me semble-t-il, à induire une lecture ontologique des «états » auxquels donne accès le respect de la loi : telle est peut-être la raison du fonctionnement substantiel de la pureté (effet du respect de la loi, mais aussi vulnérable à la contagion et parfois perdue en l'absence de toute intentionnalité). Quant à l'impureté, en partie conditionnée par des actes volontaires pour les Hébreux, elle peut être conçue comme une propriété constante (ontologique) des autres peuples. En d'autres termes, ces derniers sont à des degrés divers privés de l'accès à des "états " qui combinent de façon inextricable l'inné et l'acquis : impossible ici de trancher entre une nature (faite de caractères hérités) et une culture marquée par la reconnaissance de règles spécifiques. La forme de l'Alliance qu'institue la circoncision illustre bien le croisement des deux séries. Tout en étant une marque corporelle qui renforce artificiellement la discontinuité des physicalités entre les hommes, elle est toujours traitée à la fois comme une obligation (en tant que contrepartie de l'Alliance) et, selon qu'elle est ou non pratiquée par un peuple, comme une de ses propriétés objectives, nécessairement corrélée à sa position généalogique dans l'ethnogenèse.

18. Le fait qu'il puisse l'aliéner indique bien qu'elle est traitée comme une réalité substantielle qui vient s'ajouter aux autres éléments de l'identité personnelle. À noter aussi que, selon Deutéronome $(23,8)$, l'Édomite ne doit pas être tenu pour abominable " car c'est ton frère ». Le privilège accordé dans le même texte aux Egyptiens est plus mystérieux, étant motivé par le fait qu'Israël «a été étranger chez eux". 
D'où la casuistique complexe qu'ouvrent les conversions (ou intégrations) de membres d'autres peuples ainsi que les "grands acteurs » issus de lignées mixtes : Jephté, par exemple, fils d'un Hébreu et d'une "prostituée étrangère ", voire même le roi David, qui compte dans son ascendance Ruth la Moabite ${ }^{19}$. Mais cela nous conduit déjà à un autre versant de cette analyse : la prise en compte des individus dans leur rapport au divin.

\section{États de puretélimpureté et composants de la personne}

Il résulte de ce qui précède que chaque homme pris individuellement peut être identifié par un faisceau de caractères - généalogie, aînesse, identité ethnique, appartenance à telle tribu, avec les rapports à la loi que chacun de ces composants suppose - qui tendent vers une individuation assez poussée, même si celle-ci n'est relevée que pour les personnages les plus en vue - Grands Prêtres, Juges et bientôt rois. Dans ce dispositif, l'ancestralité joue un rôle essentiel en tant que pourvoyeuse de divers constituants de l'identité des personnes. Aussi l'absence de tout culte des ancêtres, relevée par Mary Douglas comme un signe de singularité de la religion instituée par le Lévitique, doit-elle être relativisée : comme c'est souvent le cas selon Philippe Descola dans les ontologies analogistes, les ancêtres sont bien présents dans l'horizon intellectuel du texte biblique, même si - monothéisme oblige - ils ne font l'objet d'aucun culte organisé. Il reste à se demander si le versant "volontariste» de la conformité à la loi que suppose le respect des différentes strates de l'Alliance peut être lui aussi analysé dans un sens ontologique lorsqu'on considère les seuls Hébreux. Ce point n'est peut-être pas nécessaire à ma démonstration, mais il permettrait de renforcer la ressemblance déjà bien établie entre l'ontologie biblique et le modèle analogiste.

Notons pour commencer que des différences individuelles sur le plan des physicalités viennent s'ajouter au répertoire invoqué ci-dessus: "L'homme aux testicules écrasés ou à la verge coupée ne sera pas admis à l'assemblée de Yahvé », lit-on dans le Deutéronome (23, 2). Ces critères d'intégrité physique sont encore plus importants dans le cas du Grand Prêtre. Du côté des intériorités, le meilleur candidat à une évaluation différentielle de l'état des personnes reste la pureté et/ou la sainteté. Si je lis bien les textes, la pureté se présente comme un aspect seulement de la sainteté, quant à elle conditionnée par l'obéissance à l'ensemble des lois du Lévitique, qui concernent aussi la justice, les obligations cultuelles, etc. Sous un aspect plus ontologique, des degrés de sainteté semblent également relever de la position généalogique, comme on le voit avec les Lévites

19. Voir sur ces questions «La légitimité de Salomon », dans Leach (1980 : 161-221). 
et les Grands Prêtres. On l'a déjà noté, positions et obligations se rejoignent dans les règles de pureté plus exigeantes de la caste sacerdotale.

Examinons à présent le couple pureté/impureté dans le cas des Hébreux ordinaires. On constate immédiatement que les occasions de se rendre impur sont innombrables, mais que la logique qualitative binaire présence/absence s'accompagne d'évaluations quantitatives : les infractions aux lois de pureté sont d'une gravité inégale, que traduit la gradation de leurs conséquences. Tout se passe en somme comme si chaque loi instituait son registre de puretélimpureté, l'effet des transgressions étant la perte d'une sorte seulement de pureté - alimentaire, sexuelle, etc. Certes, la logique binaire réapparaît dans le rapport au culte puisque toute forme d'impureté est également invalidante. Mais la diversité des modes de récupération de la pureté et la durée inégale des états d'impureté suggèrent malgré tout une pluralité de processus et de modes d'action spécifiques : une logique seulement binaire appellerait plutôt une modalité rituelle unique de remise en conformité, comme l'absolution des péchés dans le cas du christianisme.

Il n'est donc pas invraisemblable de considérer l'impureté comme un pouvoir causal différencié à la fois qualitativement et quantitativement, capable d'affecter des dispositions elles-mêmes distinctes. Dans cette hypothèse, les différents domaines de pureté pourraient être assimilés à des propriétés constitutives de la personne susceptibles de "s'absenter" de façon plus ou moins durable et de "revenir " à l'issue de manipulations rituelles adéquates jointes à l'épuisement de l'effet causal de la source d'impureté. J'utilise à dessein un langage qui renforce la parenté entre l'anthropologie biblique et celle de systèmes analogistes analysés par Philippe Descola. Car il me semble qu'il y a bien, sur le fond, une similitude troublante entre l'Hébreu respectueux du Lévitique, toujours suspect d'avoir enfreint l'un des 613 commandements identifiés par la tradition rabbinique, et le Dogon aux prises avec le foisonnement des composants de son identité qui a pu faire douter, écrit Descola, qu'il «ait pu à tout moment conformer l'ensemble de ses actes aux préceptes régissant le bon fonctionnement de cette cosmologie surpeuplée" (2005: 313).

Un homme n'est donc pas tout à fait le même selon le degré de conformité de son essence (évalué en termes de pureté) aux attentes du divin. L'histoire de Samson offre une illustration hyperbolique de ces variations : sa force herculéenne, liée aux prescriptions spécifiques d'un nazir, est proportionnée à la longueur des cheveux que son statut lui interdit de couper. Fragilisé dans son identité de super-Hébreu par sa liaison amoureuse avec Dalila la Philistine, il parcourt tout le chemin de la parfaite pureté (et la force) à la quasi-apostasie et à l'impuissance que lui vaut un manquement objectif à la loi dont il n'est qu'indirectement responsable. 
Je pense avoir suffisamment établi que la classification biblique des animaux et des hommes ne se laisse pas réduire aux dichotomies homme/animal et nature/culture instituées par l'ontologie naturaliste. Que la complexité des humains se distingue de la relative simplicité des animaux pour ce qui concerne le nombre des composants de leur identité n'est pas une objection: tous les systèmes analogiques, selon Philippe Descola, manifestent cette propriété. L'élément significatif est plutôt, me semble-t-il, l'introduction entre les hommes de principes de différenciation similaires à ceux qui distinguent les animaux entre eux et les hommes des animaux. Cette proposition peut, bien entendu, prêter à un malentendu fâcheux : elle suppose en effet un mode de différenciation des hommes tout à fait comparable à celui qui existe entre l'homme et l'animal. Mais il serait tout à fait absurde de considérer que les auteurs de ces textes étaient " racistes " au sens moderne (c'est-à-dire : " naturaliste ") du terme. La logique analogiste des «petites différences » dans l'ordre de la création conduit nécessairement à distribuer tous les êtres, humains et non humains, dans un tableau continu de variations. Des variations qui, on va le voir, peuvent aussi se comprendre dans une perspective dynamique.

Cette remarque nous ramène ainsi, après un long détour, au point de départ de cette enquête : la formule du Lévitique "Soyez saints parce que je suis saint». L'injonction, nous le savons, concerne les seuls Hébreux. Mais elle fait plus que leur assigner une place dans l'ordre de la création, elle suggère aussi un devoir d'élévation, de dépassement d'un état statique de l'ordre cosmique. Que peut donc signifier une telle prescription, et en quoi relève-t-elle encore d'une conception analogiste du monde? C'est ce que je vais à présent essayer de clarifier.

\section{Une perfection à accomplir}

Mythologie fondatrice d'un peuple, les textes que nous étudions sont en même temps porteurs de l'étiologie de sa religion. Et de même que la naissance des peuples, jointe à la spécification du statut particulier des Hébreux, s'échelonne dans le temps, de même le contenu de l'Alliance s'enrichit au fur et à mesure jusqu'à atteindre sa forme complète avec les révélations du Sinaï. Mais, à cette étape, sa réalisation n'est encore que promesse : les Hébreux ne sont toujours pas maîtres de la Terre promise, et leur installation en ces lieux sous l'aspect d'un peuple uni dans le culte à Yahvé connaîtra encore bien des incidents de parcours. Ajoutons enfin que la réalisation du programme divin reste conditionnée par le respect de l'Alliance, une clause qui s'avère difficile à satisfaire. Ainsi, moins qu'un tableau statique de la création précisant une fois pour toutes la place et les devoirs de chacun (peuples et animaux), le Pentateuque offre 
l'image d'un processus : l'introduction - conditionnelle - dans le monde d'un ordre supérieur à l'ordre cosmique, que l'on peut définir par une ressemblance croissante entre le Créateur et la création. C'est du moins ainsi que l'on peut comprendre l'injonction divine "Soyez saints parce que je suis saint ".

Si cette hypothèse est exacte, on comprend aussi que le simple cosmos puisse impliquer l'existence d'êtres objectivement inférieurs aux autres en dignité : des animaux par essence impurs ne sont pas un scandale, comme le pense Mary Douglas, mais le signe d'un monde encore à parfaire grâce, en particulier, à l'obéissance des hommes à des lois d'Alliance de plus en plus exigeantes. Les Hébreux sont, bien entendu, les premiers concernés par ce programme. Mais, comme on l'a vu à travers la diversification hiérarchique des peuples, le respect de formes encore incomplètes de l'Alliance (contrat noachique, circoncision, pratique du sacrifice) contribue en quelque façon à augmenter le niveau global de «sainteté » du monde.

Les autres êtres vivants ont-ils une place dans ce processus de sanctification? On l'a dit, certains d'entre eux - les animaux impurs - en sont écartés. Associés seulement à l'ordre cosmique, ils n'ont d'autre destin que de rester à leur place, aussi longtemps du moins que la sanctification globale du monde n'est pas accomplie: il y a lieu de douter qu'il y ait encore des porcs dans l'univers des derniers temps. En revanche, il existe peut-être dans le présent une double voie de "promotion" pour les plantes et les animaux purs: leur usage alimentaire ou sacrificiel. Cette idée n'est pas explicitement présente, à ma connaissance, dans le texte biblique. Elle est en revanche développée dans le judaïsme actuel : l'aliment prenant les caractères du mangeur, il est "promu » ontologiquement dès lors que ce dernier est situé plus haut dans la hiérarchie des êtres. Le végétarisme, adopté par certains juifs d'aujourd'hui au nom d'une bienveillance supposée imiter celle du Créateur lui-même, serait donc un choix malheureux, limitant la sanctification objective du monde animal ${ }^{20}$.

Quant au sacrifice, son programme rituel suffit à témoigner de sa capacité d'élévation du niveau de sainteté du monde : la séparation de la chair et de l'âme-sang s'accompagne de son retour symbolique vers le Créateur lorsque le sang est répandu sur les cornes de l'autel. La vie revient ainsi vers son principe au lieu de rester mêlée à la chair du cadavre. D'une autre manière, Mary Douglas montre que le traitement du corps de la victime revient à le conformer à la tripartition hiérarchique des rapports à Dieu que traduit également la structure de la Tente de Rencontre et plus tard 
celle du Temple (parvis, saint et saint des saints) : la chair, la graisse entourant les entrailles et le sang font l'objet de traitements rituels distincts (2004: 91-112).

$\mathrm{Si}$, dans le Pentateuque, le processus de sanctification du cosmos reste incertain, il trouve en revanche une expression sans équivoque dans les textes prophétiques. Une réalisation approchée des promesses de l'Alliance a en effet eu lieu avec les règnes de David et, de façon plus ambiguë, de Salomon. Mais les catastrophes qui les suivent relancent un processus d'Alliance-promesse décalé vers le futur: Dieu n'abandonnera pas son peuple et lui offrira enfin - à la fin des temps - la vie sainte à laquelle il s'est jusqu'ici dérobé. Cette ultime étape suppose une universalisation des lois de l'Alliance, symbolisée chez certains prophètes par l'accès du peuple juif à une royauté universelle. Elle s'accompagne d'une transformation de l'ordre cosmique en un ordre saint qui concerne aussi les animaux : "Le loup habite avec l'agneau, la panthère se couche près du chevreau, veau et lionceau paissent ensemble sous la conduite d'un petit garçon. La vache et l'ourse lient amitié, leurs petits gîtent ensemble. Le lion mange de la paille comme le bœuf. Le nourrisson s'amuse sur le trou du cobra, sur le repaire de la vipère l'enfant met la main ", écrit Isaïe (11, 6-9). Osée évoque quant à lui un "pacte [d'Israël] avec les bêtes des champs, avec les oiseaux du ciel et les reptiles de la terre » $(2,20)$.

Cette évolution concerne, au moins dans le christianisme, jusqu'au monde inorganique : la "nouvelle terre", dépourvue des mouvements et profondeurs inquiétantes de la mer, unie à un nouveau ciel immobile (il n'y aura désormais plus de nuit, ni de lune, ni de soleil), réalisera un ordre situé au-delà des " séparations » qui caractérisaient le cosmos originel (Apocalypse 21).

\section{Discussion et prolongements}

Le point de vue dynamique qui a été introduit dans l'examen des effets de l'Alliance contribue à rapprocher encore le modèle biblique de l'ontologie « analogiste » définie par Philippe Descola. Il met au jour, en effet, des possibilités de circulation des composants des êtres vivants entre les différents étages de la création, dont le sacrifice offre l'expression rituelle la plus claire. Celui-ci, toutefois, est avant tout un facteur de restauration de l'ordre à courte portée temporelle lorsqu'il permet simplement à un homme de retrouver une forme de pureté perdue. En revanche, l'idée d'une sanctification progressive du monde s'inscrit dans une temporalité irréversible de longue durée qui semble plutôt caractéristique des seules cosmologie et eschatologie judéo-chrétiennes. Ce point mérite un commentaire. 


\section{Analogisme et eschatologie}

Mon propos n'est en aucune façon de nier la singularité de la pensée biblique ou au contraire de la déclarer irréductible. Mais la singularité n'exclut pas l'appartenance à des classes plus larges. La cosmologie biblique pourrait donc être un exemple, peut-être unique, de rencontre entre une ontologie analogiste et une conception linéaire et orientée du temps. Une conséquence de cette convergence est que les relations entre humains et non-humains (animaux ou êtres surnaturels) se trouvent elles-mêmes soumises à des conditions temporelles. Leur forme dominante dans l'histoire des hommes est la séparation : il n'y a pas plus de dialogue ordinaire entre l'homme et les animaux qu'entre l'homme et Dieu. À l'inverse, cette communication est possible aux deux extrémités de la chaîne temporelle.

Cette distribution dans le temps des hypothèses ontologiques permet peut-être de rendre compte des aspects "animistes» de certains des textes que nous avons rencontrés. Bien entendu, il pourrait s'agir simplement de "résidus" d'un autre âge, ou encore de manifestations de l'activité de ce schème de construction qui, s'il est comme le suggère Descola inné et universel, aurait joué à la marge sans remettre en cause la prépondérance de l'ontologie analogiste. Une autre explication me paraît cependant plus convaincante. L'animisme suppose une synchronie entre le monde des hommes et celui où les animaux vivent comme des hommes. Le témoignage des chamanes et les rêves des humains ordinaires viennent confirmer la coexistence de ces univers et la réalité actuelle des interférences de leurs pouvoirs causaux. À l'inverse, le texte de Gn3 comme le discours prophétique marquent la distinction entre un avant et un après. L'hypothèse animiste d'une similitude des intériorités humaines et animales relève ainsi d'une perfection de l'ordre de la création distincte de l'ordre cosmique dans lequel s'inscrit l'existence des hommes (du moins des hommes ordinaires). Pendant la durée de l'histoire humaine, cette similitude des intériorités se dissout, et c'est l'analogisme qui décrit le mieux les différences et continuités entre les humains et les non-humains. Mais la rupture de la faute ne renvoie pas l'animalité dans une altérité radicale par rapport à l'humanité : l'intégration des animaux à l'Alliance en témoigne, sans impliquer pour autant une similitude des animaux et de l'homme sur le plan des intériorités. De même, la sanctification ultime du monde ne remet pas en cause les hiérarchies actuelles. Le texte d'Isaïe cité plus haut donne plutôt à penser que les animaux terrestres dans leur ensemble entrent désormais dans la classe du bétail ou des animaux familiers et restent en conséquence subordonnés à un homme redevenu innocent (d'où l'image de l'enfant-pasteur). 
Une interprétation "naturaliste» de la situation intermédiaire rendrait donc également incompréhensibles le point de départ et le point d'arrivée de l'histoire du monde: pourquoi tant d'animaux au jardin d'Éden et à la fin des temps? Si l'eschatologie biblique était strictement anthropocentrique (et donc, à certains égards, "naturaliste»), leur présence serait tout à fait superflue. De façon symétrique, une lecture "animiste" se heurte cette fois à la double distance des intériorités et des physicalités qui caractérise le régime intermédiaire des relations homme/animal. Concrètement, cela signifie que la communication entre eux est rompue sans que pour autant leur discontinuité soit totale et définitive. La même remarque vaut pour les relations des hommes avec ces non-humains que sont les anges ou Dieu en personne. Mais il y a aussi des exceptions qui méritent d'être analysées : toutes les scènes qui, entre l'expulsion du jardin d'Éden et la fin des temps, décrivent un dialogue homme-Dieu ainsi que les occurrences, plus rares, de relations du même ordre des hommes et des animaux.

Certes, les mythes du monde entier nous ont habitués à des récits où animaux, hommes et divinités interagissent sur la base d'une commune intériorité de type humain. Eux aussi, le plus souvent, placent ces situations en un temps d'avant le temps et pensent par contraste l'état actuel du monde et de l'humanité. C'est là la situation du mythe édénique dans la Bible, auquel s'ajoute en miroir l'utopie des Temps derniers. L'ontologie animiste, en particulier à travers ses expressions perspectivistes, est sans doute celle qui se distingue le plus nettement de ce schéma en conservant la synchronie de ce qui est ailleurs situé dans une série temporelle. Le naturalisme, quant à lui, éternise la figure de la séparation après avoir éventuellement admis l'existence d'une bifurcation définitive entre les intériorités animale et humaine.

Le totémisme et l'analogisme entretiennent des rapports avec le temps plus ambigus qui réagissent sur les relations envisageables entre les différentes catégories d'existants. N'ayant rien trouvé dans la Bible qui évoque l'ontologie totémiste, je ne traiterai que du dernier cas. Comme on l'a dit, la Bible nous offre une lecture analogiste de l'histoire humaine encadrée par deux séquences plutôt animistes. La première scansion n'a rien d'original et n'implique pas nécessairement la vision d'un temps globalement orienté. Ce serait même plutôt le contraire si l'on suit les interprétations ordinaires des rapports des mythes d'origine aux rites qui suggèrent, quant à eux, une conception cyclique du temps. C'est donc l'eschatologie qui doit être la clé de la compréhension des rapports humains/non humains dans la phase intermédiaire et, plus précisément, des formes de communication de type "animiste " qui subsistent à ce moment-là. Mon hypothèse est que ces connexions sont rendues possibles par la sainteté plus 
élevée des hommes qui y sont impliqués : une sainteté qui ou bien perpétue l'innocence des premiers temps, ou bien anticipe sur la sanctification ultime du monde. Cela est évident dans la Bible hébraïque avec les figures du patriarche, du Juge, du roi béni et du prophète, qui tous maintiennent le contact avec le divin dans un contexte de séparation. En va-t-il de même pour le contact avec les animaux ? Loin d'être systématique, la conjonction des deux compétences est cependant bien attestée. L'épisode du déluge, par exemple, sous-entend que Noé dirige les animaux sauvages aussi bien que domestiques sans rencontrer de résistance particulière et peut donner au corbeau et à la colombe une mission qui implique de leur part une capacité de collaboration de type humain. Le rétablissement de la communication avec l'animal (et surtout l'animal sauvage) suppose en effet qu'il récupère, au moins provisoirement, une intériorité analogue à celle de l'homme. Bien des épisodes bibliques évoquent cette figure de l'animal miraculeusement soumis à un être saint : Élisée mobilise une ourse pour punir les enfants qui se moquent de lui, Daniel est nourri par des corbeaux. L'ânesse de Balaam, quant à elle, voit l'ange de Yahvé et parle à son maître pour le faire entrer dans sa brève mission prophétique. Bien entendu, ces récits peuvent aussi se lire comme des apologues moraux, mais leur lien presque systématique avec l'action d'un être saint n'en est pas moins significatif. Ce type de motif est repris à satiété dans l'hagiographie chrétienne. J'ai pu montrer que les comportements les plus "humains " des animaux interviennent toujours dans des interactions avec une figure de saint mystique (Albert 1995). Et les hagiographes ne manquent pas d'y voir une anticipation des Temps derniers.

Il semble donc que l'ancien judaïsme, suivi en cela sur certains points par le christianisme, dispose de sa propre théorie de l'histoire des rapports de l'homme à l'animal et de leur statut ontologique respectif: l'animisme est une occasion manquée, il n'a sa place que dans un paradis perdu ou à venir. Dans l'intervalle, les relations des deux sortes de créatures sont régies par le cadre analogiste. Il revient aux règles alimentaires et au sacrifice d'exprimer dans ses formes les plus élevées la parenté dans la différence qui les caractérise. Mais ce sont aussi les deux points sur lesquels le christianisme introduit les ruptures le plus brutales avec le judaïsme. Quelles sont donc leurs conséquences sur la pensée de l'animal?

\section{Relectures juives et chrétiennes}

Je ne ferai ici qu'esquisser une étude qui mériterait à elle seule un tout autre développement. Ma faible compétence sur la tradition rabbinique me conduira à n'en retenir que deux idées, qui restent dans une large mesure à valider : 
1) Au cours de l'histoire du judaïsme ancien et diasporique, je crois apercevoir une tendance au renforcement du schème analogiste. Cela se traduit par exemple par l'introduction d'une angélologie plus ou moins complexe, qui revient à prolonger du côté du divin les strates hiérarchiques constatées dans la différenciation des peuples.

2) La permanence des interdits alimentaires (qui, précisons-le, concernent tous des produits d'origine animale), de la circoncision, de la transmission par filiation de l'appartenance religieuse offre un solide îlot de résistance aux partages dualistes promus par le naturalisme : nature/esprit ou nature/culture. Une "nature" ontologiquement porteuse de catégorisations de l'ordre du pur et de l'impur n'est pas celle du naturalisme. Il ne fait guère de doute, par ailleurs, qu'à l'intérieur du judaïsme contemporain s'immiscent également des influences de l'ontologie naturaliste dominante. Il serait sans doute intéressant de relire à la lumière de ces hypothèses certains des débats qui divisent les communautés (par exemple, la circoncision des fils de juifs mariés à une non-juive).

Du côté du christianisme, la situation est beaucoup plus confuse. Le rejet des interdits alimentaires (sauf, dans un premier temps au moins, celui qui concerne le sang) et la claire ouverture à tous les peuples de la conversion introduisent une rupture radicale sur des points sensibles de la version juive de l'ontologie analogiste. La désacralisation de la nature, l'affirmation de plus en plus nette d'une anthropologie dualiste issue du platonisme sont autant de traits qui, comme l'a noté Philippe Descola, préparent ou déjà illustrent le triomphe de l'ontologie naturaliste en Occident. Mais cette orientation n'est pas la seule. Elle se heurte bien sûr à l'interprétation de l'héritage scripturaire de l'Ancien Testament qui, si mon analyse est exacte, relève essentiellement de l'analogisme tout en offrant des supports textuels à l'animisme, voire au naturalisme au prix de quelques interprétations hâtives.

L'analogisme marque fortement la théologie chrétienne tardo-antique et médiévale jusqu'au XII ${ }^{e}$ siècle. Pour ne retenir que quelques exemples, il faut sans doute reconnaître une portée bien plus que rhétorique à l'usage envahissant que font de l'analogie aussi bien la littérature des bestiaires que la glose des textes vétérotestamentaires à travers l'allégorèse et la typologie (de Lubac 1959; Auerbach 2003). Dans tous les cas, la réalité, naturelle ou historique, est sommée d'être plus que «factuelle». En devenant une suite objectivée de prophéties et de leçons de morale ou de théologie, elle engendre un horizon d'intentionnalité qui suppose lui-même un impossible clivage entre nature, culture et histoire. Ce n'est donc pas un hasard si le «bestiaire chrétien » du haut-Moyen Âge reste largement stéréotypé et 
peu ouvert à l'observation (Voisenet 1994) : c'est que la vérité de l'animal n'est pas d'ordre "naturaliste », elle s'inscrit dans un jeu de correspondances dont l'Écriture offre les meilleurs témoignages.

Le cas des procès médiévaux d'animaux criminels, déjà évoqué en passant, semble illustrer quant à lui l'existence d'une orientation animiste. On peut assurément y voir l'influence de pensées de l'animal pour partie au moins extérieures à la tradition judéo-chrétienne (Pastoureau 2007), mais le fil d'une revalorisation sporadique des intériorités animales traverse toute la pensée chrétienne, jusqu'à la théologie de Eugen Drewermann, auteur d'un ouvrage au titre provocateur: De l'immortalité des animaux (1992). Saint Paul du reste, dans le droit fil du contrat noachique, intégrait les animaux aux perspectives du salut. Mais, du côté du catholicisme au moins, on constate plutôt, depuis le XVII siècle, des balancements entre le naturalisme et un animisme appauvri en une éthique de la compassion (Baratay 1996). Dans le contexte contemporain de forte diffusion du végétarisme (et de militantisme contre la vivisection, etc.), l'attitude chrétienne à l'égard des animaux est, plus que jamais sans doute, passée au crible. Des dizaines de sites internet, explicitement confessionnels ou animés par des militants antispécistes ou autres, se résument à des batailles de citations contradictoires et des dénonciations abruptes. La polémique ne concerne pas le seul christianisme et les positions déjà contrastées de ses diverses obédiences, mais aussi le judaïsme et l'islam. Voilà encore le sujet d'une autre étude...

Arrivé au terme de cet article, je suis partagé entre la satisfaction d'avoir trouvé du sens à des textes pour moi jusqu'ici impénétrables et l'abattement face à l'immense travail qui resterait à faire pour donner à ces aperçus l'assise scripturaire et exégétique apte à les rendre vraiment convaincants. Je n'ai à vrai dire que peu d'espoir de parvenir jamais à satisfaire aux exigences d'érudition et de compétence linguistique en vigueur dans les études bibliques. Du côté de l'anthropologie, ma situation épistémologique n'est peut-être pas bien meilleure, mais il m'est plus facile de justifier ma démarche. J'ai pris le risque, en effet, d'appliquer la théorie de Philippe Descola sans être sûr de lui être tout à fait fidèle et sans la prétention d'en donner une interprétation que j'assumerais explicitement comme la mienne. Une théorie scientifique, en sciences sociales comme ailleurs, a certes vocation à être utilisée par d'autres que son auteur sans en sortir nécessairement altérée. Mais, en l'espèce, la nouveauté et l'ampleur de l'hypothèse présentée ouvrent à coup sûr un large champ à l'incertitude ou au malentendu. Face à ces difficultés, j'ai adopté une attitude 
pragmatique et exploratoire. Je me suis contenté de tester son aptitude à éclairer des questions, à lire autrement des textes mille fois labourés. Si l'on pense qu' « il y a du vrai » dans une proposition théorique, c'est une telle intuition qu'il faut tenter de mettre à l'épreuve, au risque d'être déçu. Telle a été ma démarche dans cette étude, et la déception n'était pas au rendez-vous.

École des hautes études en sciences sociales Laboratoire interdisciplinaire Solidarités, sociétés, territoires (Université Le Mirail Toulouse 2), Toulouse albert@ehess.fr

MOTS CLÉSIKEYWORDS: homme et animal/people and animal - analogisme/analogism judaïsme/judaism - christianisme/christianity - Bible. 


\section{Albert, Jean-Pierre}

1995 "L'ange et la bête : sur quelques motifs hagiographiques ", in Bernadette Lizet \& Georges Ravis-Giordani, eds, Des bêtes et des hommes. $118^{e}$ Congrès des Sociétés historiques et scientifiques, Pau, 25-29 octobre 1993. Paris, Éd. du CTHS : 245-253.

\section{Auerbach, Erich}

2003 Figura. La Loi juive et la Promesse chrétienne. Paris, Macula.

\section{Baratay, Éric}

1996 L'Église et l'animal (France, $X V I I^{e}-X X^{e}$ siècle). Paris, Le Cerf.

\section{Belting, Hans}

2005 Hieronymus Bosch, "Le Jardin des délices”. Paris, Gallimard.

Descola, Philippe

2005 Par-delà nature et culture. Paris, Gallimard.

\section{Douglas, Mary}

1981 De la souillure. Essai sur les notions de pollution et de tabou. Paris, Maspéro.

2004 L'Anthropologue et la Bible.

Lecture du Lévitique. Paris, Bayard.

\section{Drewermann, Eugen}

1992 De l'immortalité des animaux. Paris, Le Cerf.
Finkelstein, Israël \& Neil Asher Silberman

2002 La Bible dévoilée. Les nouvelles révélations de l'archéologie. Paris, Bayard.

\section{Gibert, Pierre}

1986 Bible, mythes et récits de commencement. Paris, Le Seuil.

\section{Graves, Robert \& Raphael Patai}

1987 Les Mythes hébreux. Paris, Fayard.

\section{Leach, Edmund R.}

1980 L'Unité de l'homme, et autres essais.

Paris, Gallimard.

Lubac, Henry de

1959 Exégèse médiévale. Les quatre sens de l'Écriture. Paris, Aubier, 2 vol.

Pastoureau, Michel

2007 L'Ours. Histoire d'un roi déchu.

Paris, Le Seuil.

\section{Sainte Bible}

1961 La Sainte Bible. Éd. de l'École biblique de Jérusalem. Paris, Le Cerf.

\section{Voisenet, Jacques}

1994 Bestiaire chrétien. L'imagerie animale des auteurs du Haut-Moyen Âge ( $V^{e}-X I^{e}$ s.). Préface de Pierre Bonnassie. Toulouse, Presses universitaires du Mirail. 
Jean-Pierre Albert, Les animaux, les hommes et l'Alliance: une lecture anthropologique de quelques textes bibliques. - Cet article est un essai d'application au texte biblique des hypothèses développées par Philippe Descola dans Par-delà nature et culture. En examinant les passages consacrés aux différences et similitudes entre les hommes et les animaux et en cherchant à comprendre pourquoi les animaux sont inclus dans l'alliance entre Dieu et Noé qui suit le déluge, on établit qu'il y a moins une rupture qu'une continuité entre ces deux classes d'êtres. Un même principe hiérarchique concerne les animaux entre eux, les hommes et les animaux et enfin les hommes entre eux. Ce principe peut être mis en relation avec une théorie des composants de l'être vivant et de la personne qui est également typique de ce que Descola appelle l'ontologie analogiste.
Jean-Pierre Albert, Animals, People and the Covenant: An Anthropological Interpretation of a Few Biblical Texts. - The hypotheses developed by Philippe Descola in Par-delà nature et culture are applied to texts from the Bible. By examining passages about the differences and similarities between people and animals, and trying to understand why the latter are included in the Covenant between God and Noah after the Flood, we notice less a rupture than a continuity between these two classes of beings. The same hierarchical principle applies to animals, to people, and to people and animals together. This principle can be connected with a theory of living beings and persons, which is typical of what Descola has called an " analogistic ontology". 